\title{
Dense-plasma-driven ultrafast formation of FePt organization on silicon substrate
}

\author{
ROHIT MEDWAL ${ }^{1,3, *}$, NEERU SEHDEV ${ }^{2}$, WANG YING ${ }^{3}$, R S RAWAT ${ }^{3}$ and S ANNAPOORNI ${ }^{2}$ \\ ${ }^{1}$ Kyushu Institute of Technology, Iizuka, Fukuoka 8208502, Japan \\ ${ }^{2}$ Department of Physics and Astrophysics, University of Delhi, Delhi 110007, India \\ ${ }^{3}$ Natural Sciences and Science Education, National Institute of Education, Nanyang Technological University, \\ Singapore 637616, Singapore
}

MS received 12 March 2016; accepted 20 June 2016

\begin{abstract}
This article demonstrates the removal of organic capping and promotion of long-range 2D organization of chemically synthesized FePt nanoparticles dispersed on $\mathrm{Si}\langle 100\rangle$ substrate by means of pulsed $\mathrm{H}^{+}$energetic ion irradiation using a dense plasma focus (DPF) device. The irradiation of energetic $\mathrm{H}^{+}$ions on FePt nanoparticles clearly resulted in enhanced structural and magnetic behaviour of the FePt nanoparticles as a function of plasma focused irradiation shots. Transmission electron microscopy (TEM)/scanning electron microscopy (SEM) images of the FePt nanoparticles clearly show a marked enhancement in average particle size from $2.5 \mathrm{~nm}$ for nonirradiated sample to about $28 \mathrm{~nm}$ for four plasma focus shots irradiation. The gradual removal of organic capping over chemically synthesized FePt nanoparticles with increasing plasma focus shots exposure is confirmed using Raman spectroscopy. A uniform 2D organization of bimetallic FePt nanoparticles over $1 \mathrm{~cm} \times 1 \mathrm{~cm}$ silicon substrate is obtained with three plasma focus shots with better magnetic properties as compared with plasma-untreated FePt nanoparticles.
\end{abstract}

Keywords. Nanostructures; chemical synthesis; magnetic materials; phase transition.

\section{Introduction}

Well-assembled FePt nanoparticles over the desired substrates can be achieved by various physical and chemical methods [1-3]. Physical methods like lithography, masking, e-beam, and imprint lithography used for nano-patterning and array formation of magnetic and non-magnetic materials are highly expensive and time-consuming methods [4-8]. The chemical-based methods are most commonly used for preparing the self-assembled FePt nanoparticles with controlled particles size of narrow size distribution using surfactants [9-13]. It is well known that as-prepared nanoparticles are in face centred cubic (FCC) phase and can be transformed to the face centred tetragonal (FCT) phase with heat treatment [14]. However, one of the issues in chemical synthesis methods is that the organic capping, like oleylamine and oleic acid, used to control the particle size also reduces long-range organization of the nanoparticles over the substrate. The organic capping can be removed by various physical and chemical processes like chemical etching, thermal degradation, sputtering and different types of plasma treatment $[15,16]$. The chemical methods involve the removal of capping using suitable solvents that can dissolve organic capping. But the formation of the $2 \mathrm{D}$ self-organization of

\footnotetext{
*Author for correspondence (rohitmedwal@gmail.com)
}

the nanoparticles is not possible with chemically cleaned magnetic nanostructures due to their high dipolar interaction between the magnetic nanoparticles. Plasma-based etching is one of the best and fastest tools to clean the organic capping of FePt nanoparticles in ambient atmosphere. In the plasma processing, the energetic ion beam has been extensively used for the modification and synthesis of nanoscale structures. The surface modifications of the nanostructures due to the ion beam can be understood in the form of their grain growth and inter-particle diffusivity. The morphological and structural changes induced by the energetic ion irradiation can be observed in many materials [17-19].

The self-organization of FePt nanoparticles $\sim 300 \mathrm{~nm}$ using the chemical co-reduction method has been reported by our group earlier [20]. The obtained organization was disturbed at elevated temperature with an increase in the particle size. However, high chemical ordering 95\% with $1 \mathrm{~T}$ coercivity is achieved [20]. In order to extend the long-range self-organization of FePt nanoparticles, Langmuir Blodgett's (LB) technique has been used with different functionalizations to achieve long-range positional ordering up to $2 \mu \mathrm{m}$ [21]. However, the LB technique is tedious and time consuming. To overcome the above-mentioned issues, the longrange positional ordering in the chemically prepared $\mathrm{FePt}$ nanoparticles has been achieved by pulsed dense hydrogen ions/plasma irradiation/processing in a dense plasma focus (DPF) device. This method is simple, fast and effective. 
The effect of varying ion dose of highly energetic $\mathrm{H}^{+}$ions by varying the number of DPF exposure shots is investigated to achieve an organized 2D assemblage of FePt nanoparticles on silicon substrate. The effect of $\mathrm{H}^{+}$ion irradiation on the removal of organic capping is also investigated in detail as a function of ion doses using SEM imaging and Raman spectroscopy. A decrease in carbon Raman peak intensities clearly indicates the removal of organic capping from FePt nanoparticles surface.

\section{Experimental}

The plasma focus device (figure 1a) is a coaxial plasma gun in which the electrical energy stored in the capacitor bank is instantly transferred across the electrode assembly inside the chamber using the fast switches [18]. High voltage is transferred from the capacitor bank to the electrodes and a plasma sheath is formed across the electrodes at its closed end. After the formation of plasma sheath, the Lorentz force accelerates the conducting plasma sheath axially towards the open end of the electrode assembly. At the top of the anode, the plasma sheath compresses and forms a dense and hot plasma pinch column. The formed plasma pinch column then breaks down due to sausage $(m=0)$ instabilities, resulting in the formation of energetic ions of the filling gas species and hot dense decaying plasma, which move towards the top of the chamber; this can be used to process the sample placed down the anode stream. The details of ions/plasma processing in plasma focus device can be found in the review paper by Rawat [22]. The energetic ions and hot dense decaying plasma interact with the exposed material surface and change its properties by means of transient thermal effects and ion implantation. The thermal transient energy may also affect the surface morphology of the dispersed colloidal solution of FePt nanoparticles on the silicon substrate prepared by chemical co-reduction. In the present case, the DPF device was operated under hydrogen gas atmosphere to give a reducing environment, keeping the substrate and anode distance fixed at $4 \mathrm{~cm}$. The dense plasma device, giving rise to the short-lived hot and dense plasma $\left(1-2 \mathrm{keV}, 10^{25}-10^{26} \mathrm{~m}^{-3}\right)$ and energetic ions of a few tens of $\mathrm{keV}$ to several $\mathrm{MeV}$ $[17-19,22]$, was used to process the chemically synthesized FePt nanoparticles on the silicon substrate. The morphological studies of chemically prepared FePt on the silicon substrate were performed using a JEOL JSM - 6700F field emission scanning electron microscope (FESEM) as a function of number of plasma focus shots. The etching behaviour of hot and dense hydrogen plasma and energetic $\mathrm{H}^{+}$ion irradiation on capped FePt nanoparticles has been demonstrated using Raman spectroscopy study. The structural and magnetic phase transitions of the organized FePt nanoparticles are studied using a Bruker Advance (D8) X-ray diffractometer (XRD) and a vibration sample magnetometer (EV9 microsence), respectively.

\section{Results and discussion}

The long-range positional ordering of the self-organized FePt nanoparticles on the desired substrate surface is very crucial for device application. Chemically prepared nanoparticles do not form the long-range positional ordering up to a few micrometres as discussed in detail in our earlier report [21]. Hence, the chemically prepared FePt nanoparticles dispersed on silicon substrate were exposed to energetic ions and hot dense decaying plasma in the DPF device to achieve the desired self-organization of FePt on silicon.

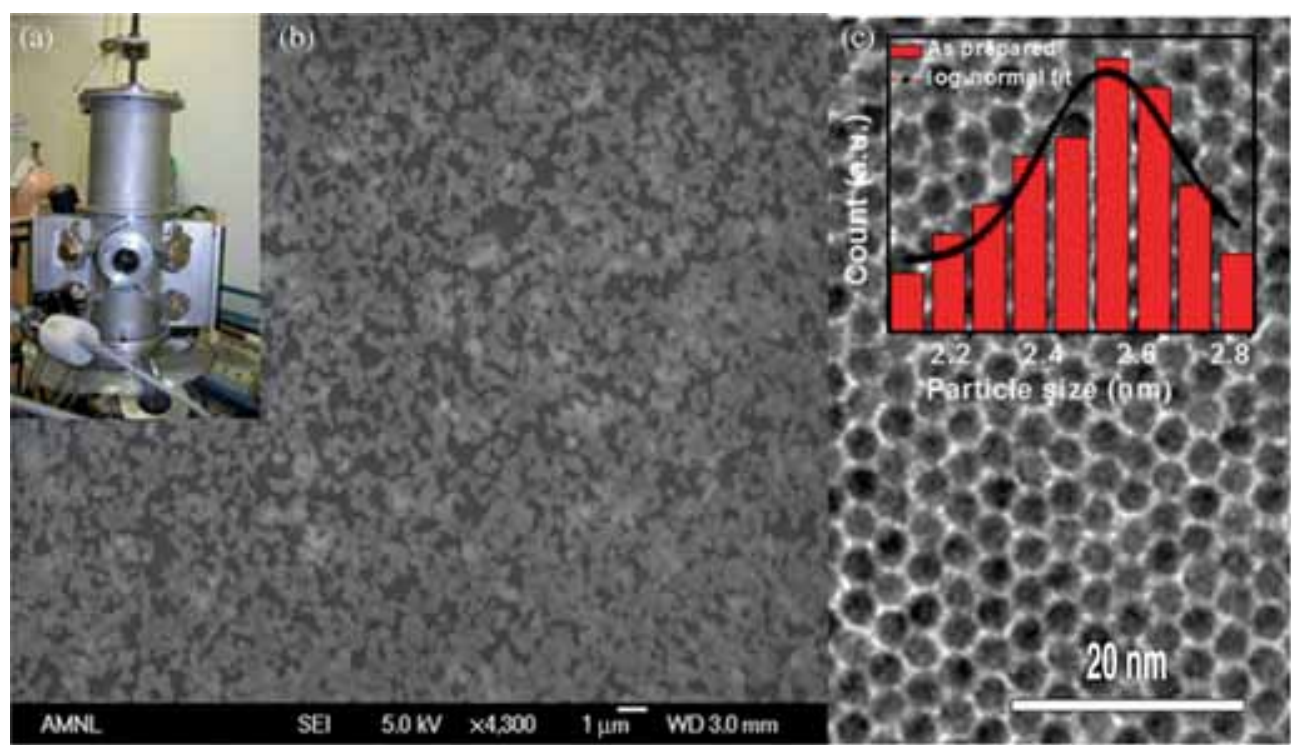

Figure 1. (a) Photographic image of the DPF instrument, (b) SEM image of as-deposited FePt nanoparticles on silicon substrates and (c) TEM image of FePt nanoparticles with the inset showing the particle size distribution. 

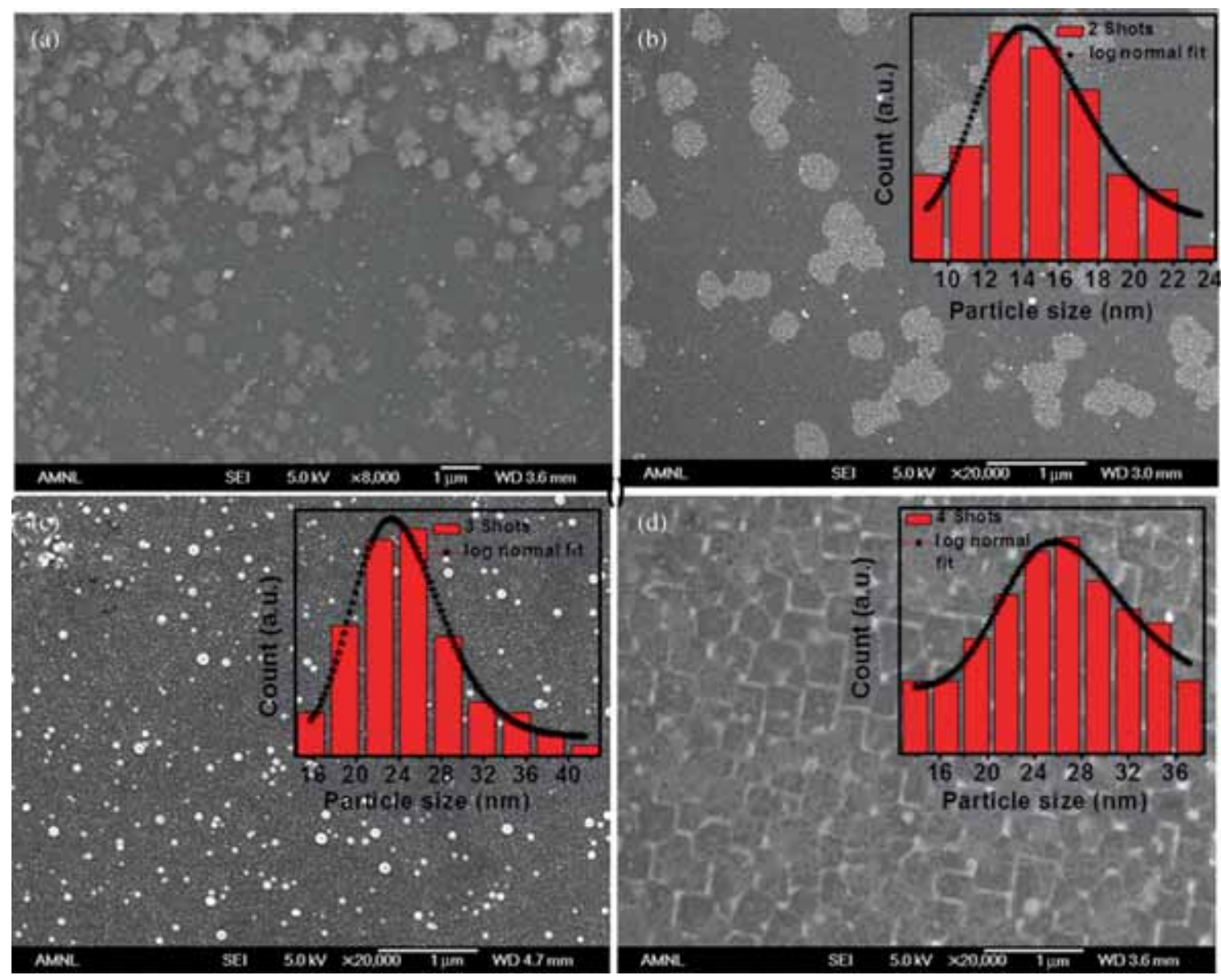

Figure 2. SEM image of chemically prepared FePt nanoparticles dispersed on silicon substrate after exposure to (a) one, (b) two, (c) three and (d) four hydrogen plasma shots. Inset of b, c and d shows the particle size distribution for two, three and four plasma shots, respectively.

Figure 1b shows the SEM image of FePt colloid on the silicon substrate and reveals the formation of globules of capped FePt nanoparticles on substrate. Such a globular morphology may be attributed to interactions among organic cappings of oleic acid and oleylamine over the nanoparticles with silicon substrates. However, long chains of the hydrocarbon over the capped nanoparticles have strong interaction among themselves, leading to the formation of the hexagonal closed packed FePt organization up to $\sim 100 \mathrm{~nm}$ as shown in transmission electron microscopy (TEM) in figure 1c. The mean size of the chemically prepared self-organized $\mathrm{FePt}$ nanoparticles has been estimated to be about $2.5 \mathrm{~nm}$ using the log normal distribution, as shown in the inset of figure 1c. The change in morphology and reduction in the clustering of organic-matrix-embedded FePt was studied as an effect of the hydrogen ions/plasma exposure shots using the DPF.

The specimen was exposed to varying hydrogen ions/plasma doses by varying number of plasma focus shots (viz. one, two, three and four plasma focus shots) keeping the distance between the source and the sample constant $(4 \mathrm{~cm})$. Hydrogen was chosen to create a reducing atmosphere to avoid further oxidation of the bimetallic FePt nanoparticles during high-energy plasma treatment. Moreover, hydrogen helps in the degradation in the organic capping, resulting in uncapped bimetallic FePt nanoparticles. Figure 2a-d shows the change in the organization of FePt nanoparticles on the silicon substrates with the number of plasma focus shots. One shot exposure reveals a reduction of the globules on the silicon substrate as shown in figure $2 \mathrm{a}$, though the complete breakdown of capping layer is not observed. This may be attributed to partial energy transferred from dense plasma and energetic ions to the surface of the film, which will not be sufficient for complete removal of organic capping from the surface of the nanoparticles. Hence, exposure of one shot is not enough to form organized FePt nanoparticle layer over silicon substrate. The presence of the organic-matrixembedded FePt nanoparticles on the silicon surface does not favour the estimation of the particle size of the nanoparticle after plasma exposure as distinct isolated particles are not observed very well in SEM images (figure 2a).

The sample exposed to two plasma focus shots, see figure $2 b$, shows long-range organization of the nanoparticles on the silicon substrate due to the complete etching of the organic capping by hydrogen plasma/ions exposure. The log normal distribution of particle size is shown in the inset of figure $2 b$, which shows the increase in the average particle 
size to $\sim 14 \mathrm{~nm}$. The increase in average particle size may be attributed to inter-particle diffusion due to intense transient thermal effect of high-energy ion beam and hot dense decaying plasma of dense focus plasma, which results in Ostwald ripening [23]. The formation of a bilayer was also observed, which is ascribed to high concentration of $\mathrm{FePt}$ nanoparticles in the particular area. Exposure to three plasma shots of the samples results in further enhancement of particle size, with average particle size of $24 \mathrm{~nm}$ and uniform arrangement of the FePt nanoparticles on the silicon surface with large positional ordering as shown in figure 2c. However, particles of bigger size with very bright contrast are also observed for three shots irradiation. These large particles are attributed to the copper etched out from the copper anode of the plasma focus device during the plasma shots by the energetic electron beam, which moves towards the anode, and also hot dense pinch plasma interaction [22]. Figure 2d shows the cracks formation on the specimen exposed to four plasma focus shots, which can be attributed to high energy transferred to this sample. The average size of FePt nanoparticles is further increased to about $28 \mathrm{~nm}$ after exposure to four plasma focus shots, as shown in the inset of figure $2 \mathrm{~d}$, due to enhanced Ostwald ripening. Clusters with large particle size were also observed on the surface at different places, indicating the agglomerations of $\mathrm{FePt}$ nanoparticles due to higher total incident energy.

In order to see the effect of hydrogen plasma on the organic capping, Raman spectroscopy as a function of plasma focus irradiation shots on FePt nanoparticles was performed. As-deposited nanoparticles capped with oleic acid and oleylamine do not show Raman spectra as reported earlier [15]. The nanoparticles exposed to one shot of hydrogen plasma exhibit the formation of the carbonaceous layer with $\mathrm{G}$ band centred at $1600 \mathrm{~cm}^{-1}$ with an evolution of $\mathrm{D}$ band at $1360 \mathrm{~cm}^{-1}$, as shown in figure 3a. The evolution of $\mathrm{D}$ and $\mathrm{G}$ bands is also reported earlier by our group as a function of different annealing temperatures $[15,20]$. This confirms the graphitization of organic layer over the FePt due to high-energy hydrogen plasma/ions exposure. The reduced intensity of $1600 \mathrm{~cm}^{-1}$ Raman peak for two shots irradiation confirms the etching of carbonaceous layer with two shots of $\mathrm{H}^{+}$plasma. The intensity of the Raman peaks corresponding to $\mathrm{D}$ and $\mathrm{G}$ bands decreased significantly with three plasma shots, indicating the removal of carbonaceous layer, as seen in figure $3 \mathrm{a}$. However, the sample irradiated with four plasma focus shots does not show any peaks corresponding to $\mathrm{D}$ and $\mathrm{G}$ bands, indicating the complete removal of the carbonaceous layer.

The effect of hydrogen ions/plasma on the structural transformation of $\mathrm{FePt}$ is investigated and shown in figure $3 \mathrm{~b}$. Samples exposed to one, two and three plasma focus shots reveal the presence of FCC phase. However, a shift in the (111) peak is observed for the sample irradiated with four plasma focus shots along with asymmetry in the (200) peak, with an evolved peak corresponding to (002). The induced asymmetry in the (111) peak is an indication of an induced tetragonality in the FePt unit cell for the sample exposed to
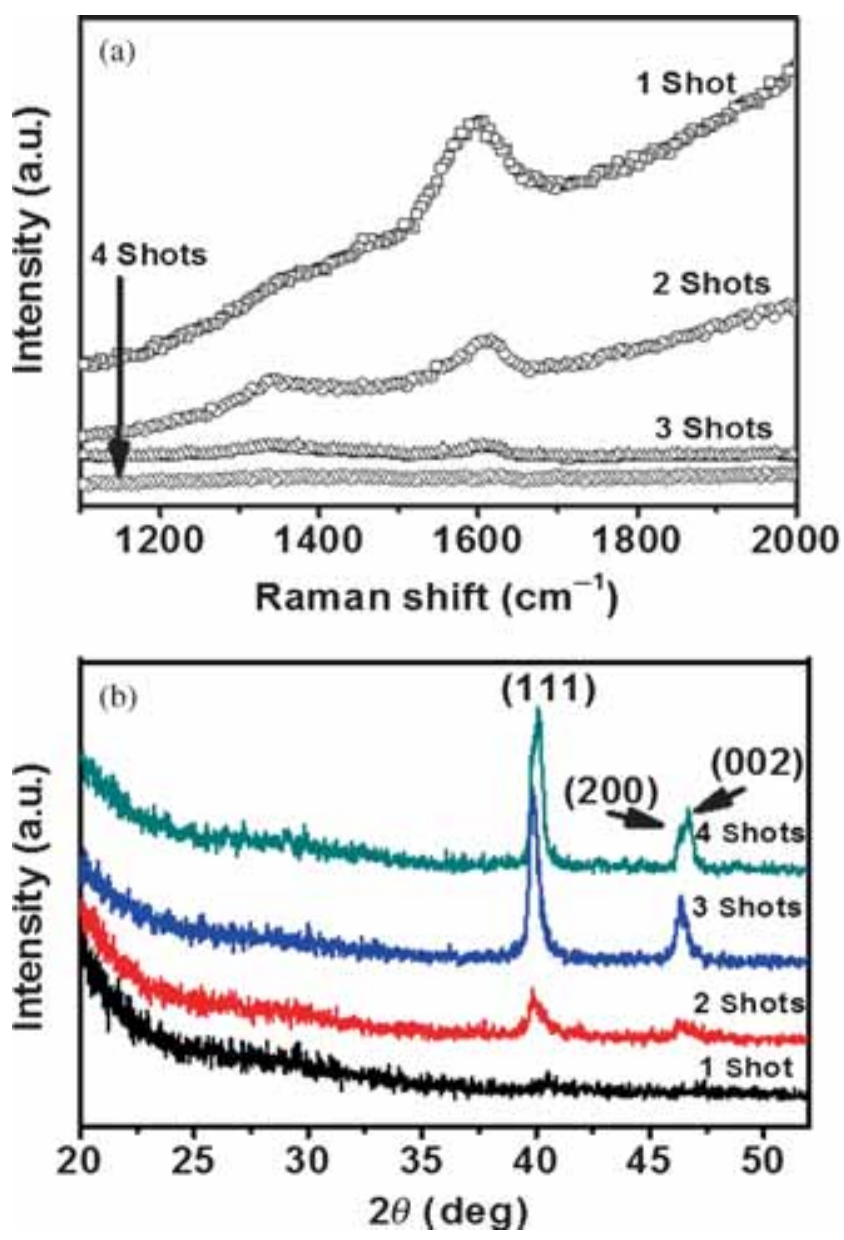

Figure 3. (a) Raman spectra and (b) XRD patterns of chemically prepared FePt nanoparticles dispersed on silicon substrate after exposing to one, two, three and four hydrogen plasma focus irradiation shots.

four DPF shots as given in figure $3 \mathrm{~b}$. This induced tetragonality will affect the magnetic properties of the FePt organization. Hence $M-H$ loops with $\pm 2.2 \mathrm{~T}$ were obtained from all hydrogen-plasma-exposed samples and are shown in figure $4 \mathrm{a}-\mathrm{f}$.

$M-H$ loop observed for one plasma shot showed soft magnetic behaviour. The loop does not tend to saturate, which may be attributed to (i) very small average particle size of about $3-5 \mathrm{~nm}$, similar to that of chemically prepared FePt nanoparticles with high surface magnetic anisotropy (figure 4a), and (ii) very weak crystallinity of this sample as observed in figure $3 \mathrm{~b}$. The samples exposed to two and three plasma shots show a saturated $M-H$ loop with soft magnetic behaviour, as shown in figure $4 \mathrm{~b}$ and c, respectively. The enhanced particle size with particle diameter of 14 and $23 \mathrm{~nm}$ for two and three plasma shots, respectively, along with improved crystallinity in FCC phase, can be attributed to the soft saturated $M-H$ loop. However, with four plasma shots, a clear bifurcation in the $M-H$ loop with improved coercivity of $\sim 500 \mathrm{Oe}$ is observed, indicating the partial transformation from soft magnetic to hard magnetic phase, 

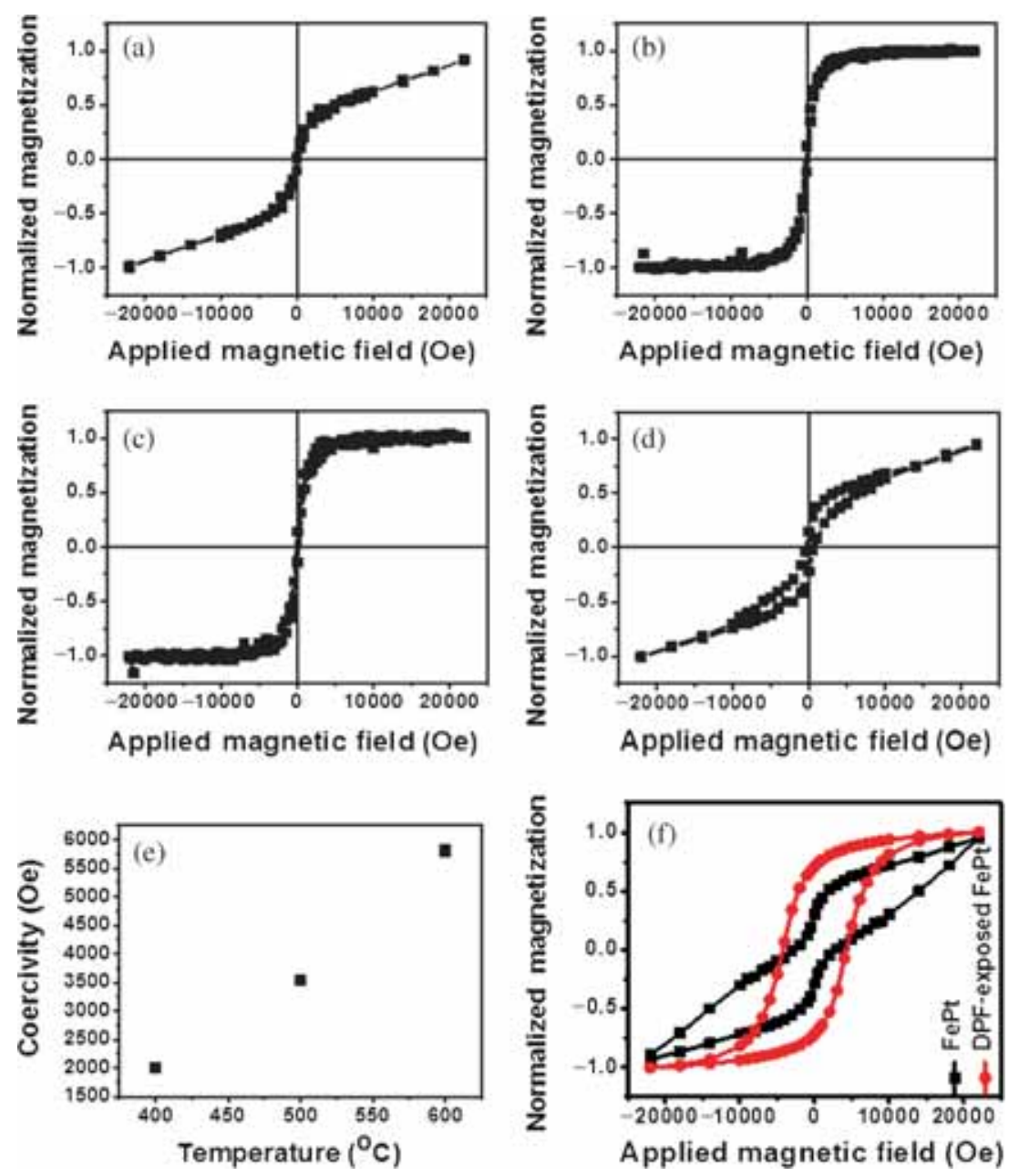

Figure 4. $M-H$ loop of chemically prepared FePt nanoparticles dispersed on silicon substrate after exposure to (a) one, (b) two, (c) three and (d) four hydrogen plasma shots, (e) coercivity as a function of post-annealing temperature for 3 shots irradiation and (f) $M-H$ loop for $600^{\circ} \mathrm{C}$-annealed FePt with and without DPF exposure.

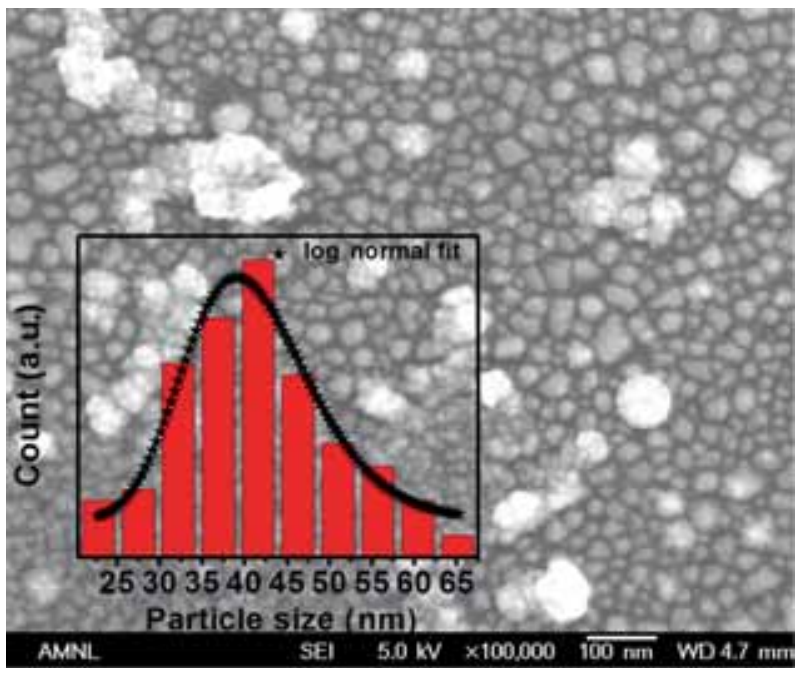

Figure 5. SEM image of the $600^{\circ} \mathrm{C}$-annealed FePt sample exposed to three plasma shots with the inset showing log normal particle size distribution. which is in agreement with XRD results (figure 4d) and can also can be attributed to increased average particle size of about $28 \mathrm{~nm}$. Further, magnetic measurement as a function of annealing temperature has been carried out on the sample exposed to three plasma shots, for which a uniform assembly of nanoparticles is obtained. A plot of coercivity as a function of annealing temperature is given in figure 4e. For FePt assemblage obtained after three plasma shots, onset of magnetic phase transformation with a coercivity of $2 \mathrm{kOe}$ is observed at $400^{\circ} \mathrm{C}$ annealing temperature. However, at elevated annealing temperature, coercivity increases to $5.8 \mathrm{kOe}$ at $600^{\circ} \mathrm{C}$, confirming the formation of hard magnetic phase as shown in figure 4e. In order to compare the effect of plasma/ion exposure on the magnetic properties, the $M-H$ loops for plasma-focus-exposed (three shots) post-annealed at $600^{\circ} \mathrm{C}$ sample and unexposed post-annealed at $600^{\circ} \mathrm{C}$ sample were recorded and are shown in figure 4f. A marked improvement in the squareness and coercivity is observed for the plasma-focus-exposed sample as compared with non-exposed FePt sample annealed at $600^{\circ} \mathrm{C}$. The sample not treated with energetic hydrogen plasma/ions shows the 
formation of $5 \mathrm{~nm} \mathrm{FePt}$ nanoparticles after annealing at $600^{\circ} \mathrm{C}[20]$. However, for the plasma-focus-exposed sample the mean diameter of the nanoparticles is $40 \mathrm{~nm}$ after annealing at $600^{\circ} \mathrm{C}$, as estimated using the SEM image shown in figure 5. The inset of figure 5 shows the log normal particle size distribution. The hydrogen plasma focus exposure promotes the removal of organic capping from the surface of the nanoparticles and increasing the coalescing process at elevated temperature, resulting in the larger particle size. Hence, large grain size with removal of organic capping from the surface reduces the surface disorderness of the magnetic crystal of FePt nanoparticles, resulting in enhanced magnetic properties of the nanoparticles.

\section{Conclusions}

The plasma treatment is very efficient for cleaning of 2D selforganization of the nanoparticles. For DC plasma, the duration of the plasma treatments varies from 20 to $60 \mathrm{~min}$ with high power; however, for the method used in this paper using the pulsed plasma focus device the total plasma/ion treatment duration is on the order of several microseconds only in each plasma focus shot. High energy density of the DPF results in the uniform distribution of the inter-particle diffusivity and grain growth, resulting in long-range positional ordering of the FePt nanoparticles on the silicon substrate surface. The pulsed energetic ion/plasma treatment using the DPF device also removed the capping layer of chemically synthesized FePt nanoparticles, while preserving the long-range ordering. Hence, the DPF device provides an excellent way of processing the chemically synthesized organically capped FePt nanoparticles to form long-range 2D organization with improved magnetic properties on the desired substrate.

\section{Acknowledgement}

This research was supported by NIE/NTU AcRF grant (RI 7/11 RSR) provided by the National Institute of Education, Nanyang Technological University, Singapore.

\section{Conflict of interest}

The authors declare that they have no conflict of interest.

\section{References}

[1] Sun S, Murray C B, Weller D, Folks L and Moser A 2000 Science 2871989
[2] Nguyen H L, Howard L E M, Stinton G W, Giblin S R, Tanner B K, Terry I, Hughes A K, Ross I M, Serres A and Evans J S O 2006 J. Chem. Mater. 186414

[3] Jeyadevan B, Hobo A, Urakawa K, Chinnasamy C N, Shinoda K and Tohji K 2003 J. Appl. Phys. 937574

[4] Luong N H, Hiep V V, Hong D M, Chau N, Linh N D, Kurisu M, Anh D T K and Nakamoto G 2005 J. Magn. Magn. Mater. 290559

[5] Xu X H, Wu H S, Wang F and Li X L 2005 Thin Solid Films 472222

[6] Takahashi Y K, Ohnuma M and Hono K 2003 J. Appl. Phys. 937580

[7] Gupta R, Medwal R, Sharma P, Mahapatro A K and Annapoorni S 2013 Superlattices Microstruct. 64408

[8] Gupta R, Medwal R, Sehdev N and Annapoorni S 2013 J. Magn. Magn. Mater. 34560

[9] Bublat T and Goll D 2011 Nanotechnology 22 315301-1

[10] Qiu L J, Ding J, Adeyeye A O, Yin J H, Chen J S, Goolaup S and Singh N 2007 IEEE Trans. Magn. 432157

[11] Li G L, Leung C W, Lei Z Q, Lin K W, Lai P T and Pong P W T 2011 Thin Solid Films 5198307

[12] Kim H, Noh J S, Roh J W, Chun D W, Kim S, Jung S H, Kang H K, Jeong W Y and Lee W 2011 Nanoscale Res. Lett. 6 13-1

[13] Li G, Dong Q, Xin J, Leung C W, Lai P T, Wong Y and Pong P W T 2013 Microelectron. Eng. 110192

[14] Medwal R, Sehdev N and Annapoorni S 2012 J. Phys. D: Appl. Phys. 45 055001-1

[15] Medwal R, Sehdev N, Gupta G and Annapoorni S 2012 Appl. Phys. A 109403

[16] Antoniak C, Trunova A, Spasova M, Farle M, Wende H, Wilhelm F and Rogalev A 2008 Phys. Rev. B 78 041406

[17] Lin J J, Roshan M V, Pan Z Y, Verma R, Lee P, Springham S V, Tan T L and Rawat R S 2008 J. Phys. D: Appl. Phys. 41 135213-1

[18] Pan Z Y, Rawat R S, Roshan M V, Lin J J, Verma R, Lee P, Springham S V and Tan T L 2009 J. Phys. D: Appl. Phys. 42 175001-1

[19] Pan Z Y, Lin J J, Zhang T, Karamat S, Tan T L, Lee P, Springham S V, Ramanujan R V and Rawat R S 2009 Thin Solid Films $\mathbf{5 1 7} 2753$

[20] Medwal R, Sehdev N and Annapoorni S 2013 J. Nanopart. Res. 15 1423-1

[21] Medwal R, Gogia K, Thakar D, Vibhu V, Mohan J R, Sehdev N and Annapoorni S 2014 Surf. Coat. Technol. 258509

[22] Rawat R S 2013 IEEE Trans. Plasma Sci. 41701

[23] Tang J, Photopoulos P, Tserepi A and Tsoukalas D 2011 Nanotechnology 22 235306-1 